\title{
A role for 12R-lipoxygenase in MUC5AC expression by respiratory epithelial cells
}

\author{
Ignacio Garcia-Verdugo*,\#,ף, Fatima BenMohamed*,\#, Sonja Tattermusch",\#, \\ Dominique Leduc*,\#, Gilles Charpigny ${ }^{+}$, Michel Chignard*,\#, \\ Mario Ollero ${ }^{\S}$ and Lhousseine Touqui*,\#
}

ABSTRACT: Eicosanoids are metabolites of arachidonic acid produced by cyclooxygenases (COXs) or lipoxygenases (LOXs). They mediate inflammation and mucus secretion in chronic pulmonary inflammatory diseases. The gel-forming mucin MUC5AC is over-expressed in the airways of patients with these diseases. MUC5AC expression is mediated by an extracellular signal-regulated kinase (ERK)/Sp1 dependent mechanism.

Our aim was to study the role of eicosanoids and their signalling pathways in MUC5AC expression.

Inhibitors of 12-LOX, but not those of COX, 5-LOX or 15-LOX, reduce MUC5AC expression induced by phorbol myristate acetate (PMA) in the bronchial epithelial cell line $\mathrm{NCl}-\mathrm{H} 292$. These inhibitors also abrogate the production of whole mucus by cell monolayers. Two forms of 12-LOX ( $R$ and $S$ ) exist in mammals. Using siRNAs we show that 12R-LOX but not 12S-LOX is involved in MUC5AC expression induced by PMA, lipopolysaccharide or transforming growth factor- $\alpha$. 12RLOX also participates in MUC2 and MUC5B expression, although to a lesser extent than for MUC5AC. Contrarily, 12R-LOX silencing does not modify interleukin-8 production. 12-LOX inhibitors reduce ERK activation and Sp1 translocation induced by PMA. Moreover, the 12RLOX product 12(R)-hydroxyeicosatetraenoic acid, induces MUC5AC expression, ERK activation and Sp1 translocation.

12R-LOX is involved in MUC5AC expression. This occurs via ERK- and Sp1-signalling pathways.

KEYWORDS: Eicosanoids, lipoxygenase, MUC5AC, mucins

$\mathrm{M}$ ucins, the major proteins within the mucus, are highly glycosylated and consist of a protein backbone with a central domain (mucin domain) susceptible to $\mathrm{O}$ glycosylation [1]. Gel-forming mucins polymerise through their cysteine-rich terminal domains and are secreted in the extracellular media. In the airways of adults, the most representative gelforming mucins are MUC5AC, MUC5B and, to a lesser extent, MUC2 [2]. These mucins are clustered on the p15 arm of chromosome 11 [3] and they display common and different regulatory mechanisms of expression [4,5]. MUC5AC is the most abundant gel-forming mucin present at the airway surface [6]. Its mechanisms of expression have been studied extensively, using the cell line NCI-H292 as a model $[4,7,8]$. Several studies from NADEL and co-workers $[8,9]$ have shown that phorbol myristate acetate (PMA) induces a matrix metalloprotease-mediated release of transforming growth factor- $\alpha$ (TGF- $\alpha$ ), which subsequently binds and activates the epidermal growth factor receptor (EGFR) to induce MUC5AC synthesis and secretion. Phosphorylation of EGFR is followed by activation of the extracellular signal-regulated kinase (ERK)/Sp1 signalling pathway [4, 7]. EGFR participates in MUC5AC release induced by other pro-inflammatory stimuli like neutrophil elastase and bacterial lipopolysaccharide (LPS) [10].

Cytosolic phospholipase $\mathrm{A}_{2}\left(\mathrm{CPLA}_{2}\right)$ is a key enzyme controlling the release of arachidonic acid (AA). Once AA is released it is further metabolised by cyclooxygenases (COXs) and/or lipoxygenases (LOXs) to generate eicosanoids. Although early studies showed that eicosanoids regulate mucin production [11, 12], the underlying mechanisms remain unknown. Recently, we have shown a role of $\mathrm{CPLA}_{2}$ in mucus hypersecretion in a mouse model of cystic fibrosis (CF) [13]. COX catalyses the biosynthesis of prostaglandins such as prostaglandin $E_{2}\left(P_{G E}\right)$. Both inhibitory and stimulatory
AFFILIATIONS

*Unité de Défense Innée et Inflammation, Institut Pasteur \#INSERM U874,

- Université Paris Diderot, Sorbonne Paris Cité (Cellule Pasteur), §INSERM U806, Hôpital Necker, Paris, and

+Unité Biologie du Développement et Reproduction, INRA, Jouy en Josas, France.

\section{CORRESPONDENCE}

I. Garcia-Verdugo

Unité de Défense Innée et Inflammation Institut Pasteur 25 rue Dr Roux 75015 Paris

France

E-mail: ignacio.garcia-verdugo@ pasteur.fr

Received:

Feb 082011

Accepted after revision:

Feb 122012

First published online:

March 222012 
effects of $\mathrm{PGE}_{2}$ on mucin secretion have been reported [12, 14]. More recently, two independent laboratories have shown a role for COX-2 in interleukin (IL)-1 $\beta$-induced MUC5AC production $[15,16]$. Conversely, LOXs are dioxygenases that catalyse the addition of oxygen to AA, yielding hydroperoxyl derivatives including hydroperoxyeicosatetraenoic acids (HpETEs) and the reduced form, HETE. Mammalian LOXs are traditionally classified as 5-, 8-, 12- or 15-LOX, according to the site of oxygen insertion within AA. In humans there are two different 12-LOXs, the platelet-type, also called 12S-LOX (gene ALOX12), and the epidermal-type termed 12R-LOX (gene ALOX12B). Although both LOXs generate 12-HETE, the 12S-LOX enzyme produces the 12(S)-HETE enantiomer, whereas the 12R-LOX enzyme produces almost exclusively the 12(R)-HETE enantiomer, representing the only mammalian LOX that directs molecular oxygen into the R position of AA [17].

12S-LOX mediates a major pathway in the metabolism of AA on platelet activation [18]. OwENS et al. [19] have found an increased expression of 12-LOX in CF patients compared with controls in both the airways epithelium and submucosal glands, but the physiological relevance of 12-LOX overexpression has not been established. The 12R-LOX pathway plays an important role in the establishment of the epidermal barrier function [20]. Conversely, 15-LOX-1 is involved in inflammatory diseases, including atherosclerosis, cancer, osteoporosis, angiotensin II-dependent hypertension and diabetes [21]. Recently, 15-LOX-1 has been implicated in MUC5AC overexpression in asthma [22].

In chronic inflammatory diseases of the airway, such as chronic obstructive pulmonary disease, asthma and CF, exacerbated production of mucus contributes to airway obstruction, favouring bacteria colonisation. Although therapies have been proposed to treat mucus hypersecretion, effective treatments are still lacking. Improving the understanding of the mechanisms of mucus production and secretion will help in the design of new pharmacological approaches in the treatment of mucus hypersecretion. In this paper, using pharmacological and siRNA approaches, we demonstrate a role for 12R-LOX in mucin expression and, particularly, MUC5AC. Induction of MUC5AC expression by 12R-LOX occurs via ERK and Sp1 signalling pathways. We propose for the first time a role of 12R-LOX in chronic pulmonary inflammatory diseases.

\section{MATERIALS AND METHODS}

\section{Cell culture and stimulation of $\mathrm{NCl}-\mathrm{H} 292$}

NCI-H292 cells obtained from ATCC (Molsheim, France), were cultured as described [13]. After $24 \mathrm{~h}$ of culture under serumfree conditions, cells were stimulated with PMA (20 nM), LPS $\left(10 \mu \mathrm{g} \cdot \mathrm{mL}^{-1}\right)$ or TGF- $\alpha\left(20 \mathrm{ng} \cdot \mathrm{mL}^{-1}\right)$ in serum-free culture media, except for LPS, to which $1 \%$ of serum was added. 12-HETE was dried from ethanolic solutions and added to the cells. For inhibition studies cells were pretreated with the indicated drugs for $1 \mathrm{~h}$ before addition of PMA and inhibitor. Drugs used in the study were tested in a wide range of concentrations $(0.1-50 \mu \mathrm{M})$ following other studies previously published [15, 16, 22]. Effects of drugs on MUC5AC expression and cell viability were measured in parallel. Concentrations presented in the study for 12-LOX inhibitors (baicalein, cinnamyl-3,4-dihydroxy- $\alpha$-cyanocinnamate (CDC)) showed an optimal inhibition of MUC5AC expression and no toxicity. Dose-response effects were also observed (data not shown). All control conditions included corresponding vehicles at the appropriate concentrations (ethanol for nordihydroguaiaretic acid (NDGA) and dimethylsulphoxide for PMA and other inhibitors). After $24 \mathrm{~h}$ in the presence of stimuli/inhibitors, supernatants were collected and the cells lysed in lysis buffer (150 mM sodium phosphate, $150 \mathrm{mM} \mathrm{NaCl}, 1 \%$ (v/v) Triton X$100,1 \%(\mathrm{w} / \mathrm{v})$ deoxycholate, $\mathrm{pH} 7.2$ supplemented with protease inhibitor cocktail). Pre-cleared samples $\left(370 \times g\right.$ for $10 \mathrm{~min}$ at $4^{\circ} \mathrm{C}$ ) were stored at $-80^{\circ} \mathrm{C}$ until analysis.

\section{Cell culture and stimulation of primary human bronchial epithelial cells}

Differentiated human primary bronchial epithelial cells, MucilAir, were purchased from Epithelix (Epithelix Sarl, Geneve, Switzerland). These cells were isolated from the bronchi of healthy subjects and cultured at the air-liquid interface for 3 weeks in mucilAir culture medium (Epithelix) until differentiation. To reduce basal levels of mucin expression, cells were cultured for $48 \mathrm{~h}$ in bronchial epithelial cell basal medium (CC-3171; Lonza, Levallois-Perret, France) supplemented with antibiotics. The cells were then stimulated in the same medium with bacteria supernatants or an equivalent dose of bacterial growth medium (Luria-Bertani (LB)) and $24 \mathrm{~h}$ after RNA extractions were performed.

Bacteria supernatants of Pseudomonas aeruginosa (PAK strain) were harvested at exponential-phase growth in LB medium. After centrifugation to eliminate bacteria, supernatants and LB medium (used as control) were filtered $(0.2 \mu \mathrm{m})$ and stored at $-80^{\circ} \mathrm{C}$ until use.

\section{SiRNA experiments}

Pre-designed siRNAs from Santa Cruz Biotechnologies (Santa Cruz, CA, USA) or control siRNAs were incubated with TransIT-siQUEST transfection reagent (Mirus Bio, Madison, WI, USA) (1 $\mu \mathrm{L}$ per well) for $20 \mathrm{~min}$ at room temperature in serum-free culture media (50 $\mu \mathrm{L}$ final volume). NCI-H292 were grown until sub-confluent $(80 \%)$ and cultured in complete cell culture media $(350 \mu \mathrm{L})$ containing the appropriate siRNA (final concentration $100 \mathrm{nM}$ ). After $24 \mathrm{~h}$ of incubation, media were removed and new medium (serum-free) plus stimuli were added for a further $24 \mathrm{~h}$. Real-time (RT)-PCR and western blotting were performed to follow-up silencing at $24 \mathrm{~h}$ or $48 \mathrm{~h}$ after transfection.

\section{ELISA}

MUC5AC ELISA was performed as previously described [13, 23] using anti-MUC5AC (clone 45M1, Neomarkers, Fremont, CA, USA) and horseradish peroxidise (HRP)-conjugated goat antimouse immunoglobulin (Ig)G antibodies. Because expression of MUC5AC in NCI-H292 changes with cell passage [23] and there is no commercial standard available for human MUC5AC, its expression is represented as a -fold increase referred to basal production (control cells). Specificity of the 45M1 clone has been demonstrated [24]. Total protein from cell lysates was quantified in order to eliminate effects of the stimuli in cell proliferation.

IL-8 and PGE 2 concentrations were measured in supernatants using a human IL-8 Kit DuoSet sandwich ELISA (R\&D Systems, Abingdon, UK) and specific PGE 2 EIA (Cayman Chemicals, Ann Arbor, MI, USA), respectively. 

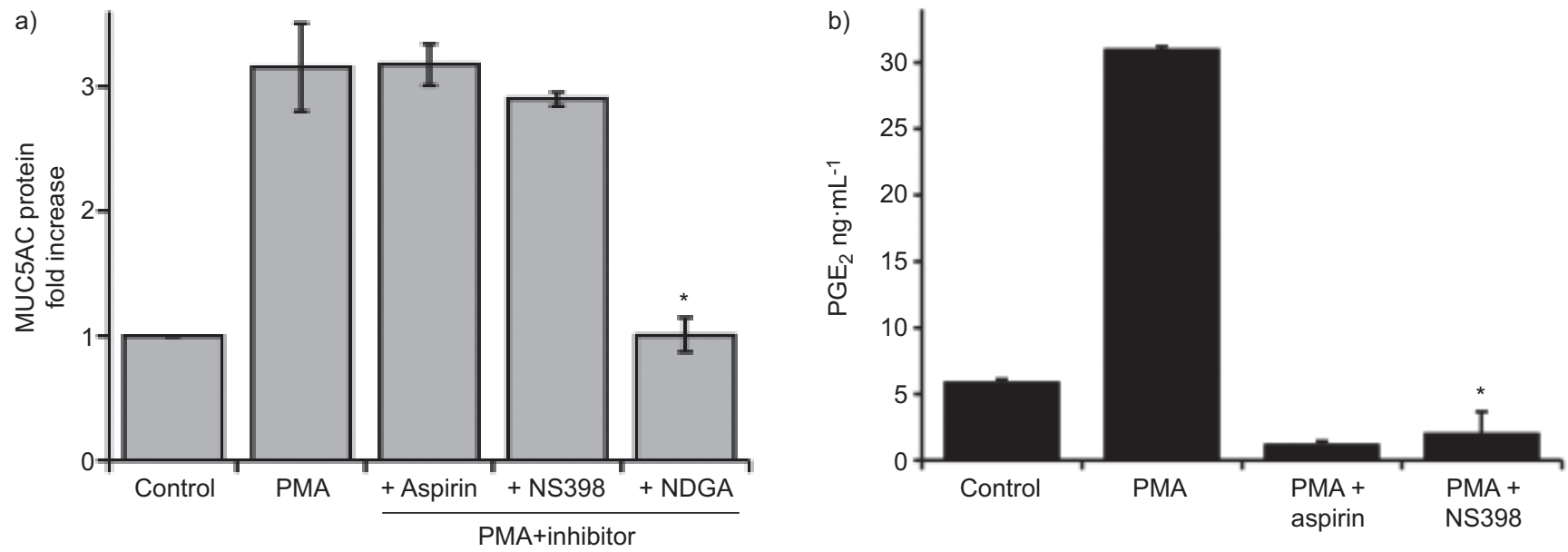

FIGURE 1. Expression of MUC5AC protein and prostaglandin $E_{2}\left(P E_{2}\right)$ secretion induced by phorbol myristate acetate (PMA) in the presence of cyclooxygenase (COX) and lipoxygenase (LOX) inhibitors. a) MUC5AC protein was measured by ELISA in NCI-H292 cell lysates after $24 \mathrm{~h}$ of stimulation with PMA (20 nM) in the presence of aspirin $(200 \mu \mathrm{M})$, NS398 $(5 \mu \mathrm{M})$ or nordihydroguaiaretic acid (NDGA) $(20 \mu \mathrm{M})$. Cells were pretreated with the indicated drugs for $1 \mathrm{~h}$ before addition of PMA. Data are represented as -fold increase referred to non-stimulated cells (control) (arbitrary units=1). Inhibitors alone did not modify MUC5AC basal secretion levels (data not shown). b) Concentration of $\mathrm{PGE}_{2}$ measured by EIA in supernatants of $\mathrm{NCl}-\mathrm{H} 292$ cells stimulated as described in a). Mean \pm SD of triplicates from three independent experiments is represented. * $\mathrm{p}<0.05$ versus PMA treatment without inhibitors.

\section{RT-PCR}

Total RNA was extracted by RNeasy Mini Kit (Qiagen, Valencia, Spain). The corresponding cDNA was synthesised using random hexamers (Roche, Boulogne-Billancour, France) and M-MLV reverse transcriptase (Promega, Charbonnièresles-Bains, France). Real-time PCR was performed in 7500 Fast Real-Time PCR System (Applied Biosystems, Carlsbad, CA, USA) using the SYBR Green Master Mix (Applied Biosystems). The primers for MUC5AC, MUC5B and MUC2 have been described previously [25]. Primers for 12R-LOX were as described by NigAm et al. [26]. Other primers were designed using the Oligo Explorer 1.1.2 software. The primers were the following: human $\beta$-actin (Fw:5'-ggacttcgagcaagagatgg-3'; Rv:5'gcagtgatctccttctgcatc- $3^{\prime}$ ), platelet-type 12-lipoxygenase (Fw:5'-ccgaggagagaagcaatacc-3'; Rv:5'-tgagggcaggaacagtgt-3'), IL-8 (Fw:5'agagacagcagagcacacaa-3'; Rw:5'-ttagcactccttggcaaaac-3'), 18sRNAr
(Fw:5' - cttagagggacaagtggcg-3'; Rw:5'- acgctgagccagtcagtgta-3'). Triplicate Ct values were analysed in Microsoft Excel (Microsoft, Issy-les Moulineaux, France) using the comparative Ct $(\Delta \Delta \mathrm{Ct})$ method as described by the manufacturer (Applied Biosystems). The amount of target $\left(2^{-\Delta \Delta C T}\right)$ was obtained as normalised to $\beta$ actin, using control cells as calibrator (arbitrary units=1) unless stated.

\section{Western blotting}

NCI-H292 cells in lysis buffer described above or radioimmunoprecipitation assay (RIPA) buffer supplemented with phosphatase-inhibitor and protease-inhibitor cocktail were electrophoresed and blotted in polyvinylidene fluoride (PVDF) membranes. Specific proteins were detected with rabbit antihuman phospho-p44/42 MAPK antibody (Cell Signaling, Danvers, MA, USA), mouse anti-human 12R-LOX antibody
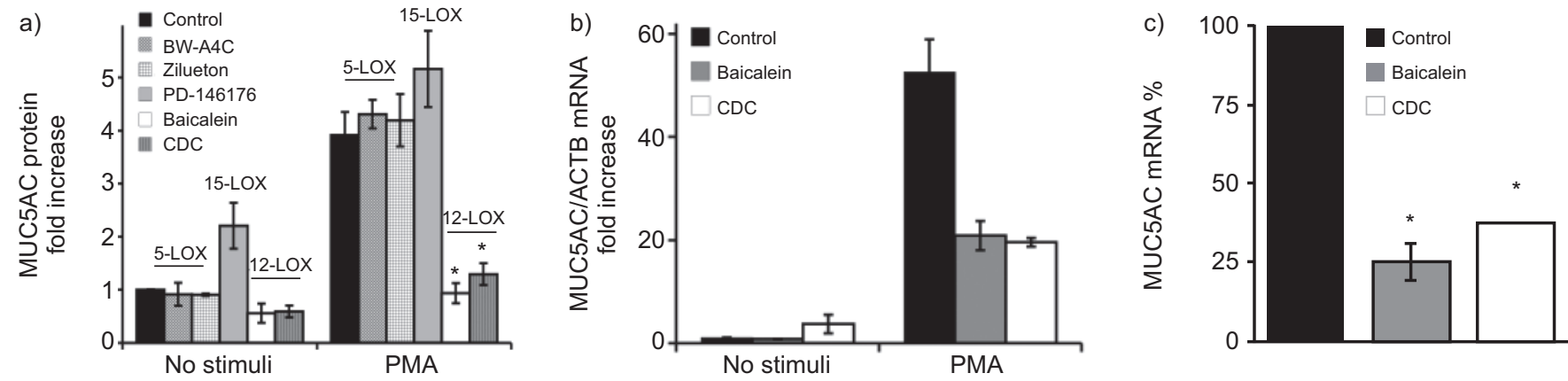

FIGURE 2. Expression of MUC5AC induced by phorbol myristate acetate (PMA) in the presence of lipoxygenase (LOX) inhibitors. a) MUC5AC protein or b) mRNA were measured in NCl-H292 cell lysates after 24 h of stimulation with PMA (20 nM) in the presence of BW-A4C, zilueton, PD-146176, baicalein (all drugs at $20 \mu \mathrm{M}$ ) or cinnamyl-3,4dihydroxy- $\alpha$-cyanocinnamate $(C D C)$ at $10 \mu \mathrm{M}$. Cells were pre-treated with the indicated drugs for $1 \mathrm{~h}$ before addition of PMA. Data are represented as -fold increase referred to non-stimulated cells (control) (arbitrary units=1). c) \% MUC5AC expression in PMA-stimulated cells referred to cells stimulated only with PMA (100\%). In a) and c) mean \pm SD of triplicates from three independent experiments is represented; in $\mathrm{b}$ ) mean $\pm \mathrm{SD}$ of triplicates from one representative experiment of a total of three is shown. *: $\mathrm{p}<0.05$ versus PMA treatment without inhibitors. 

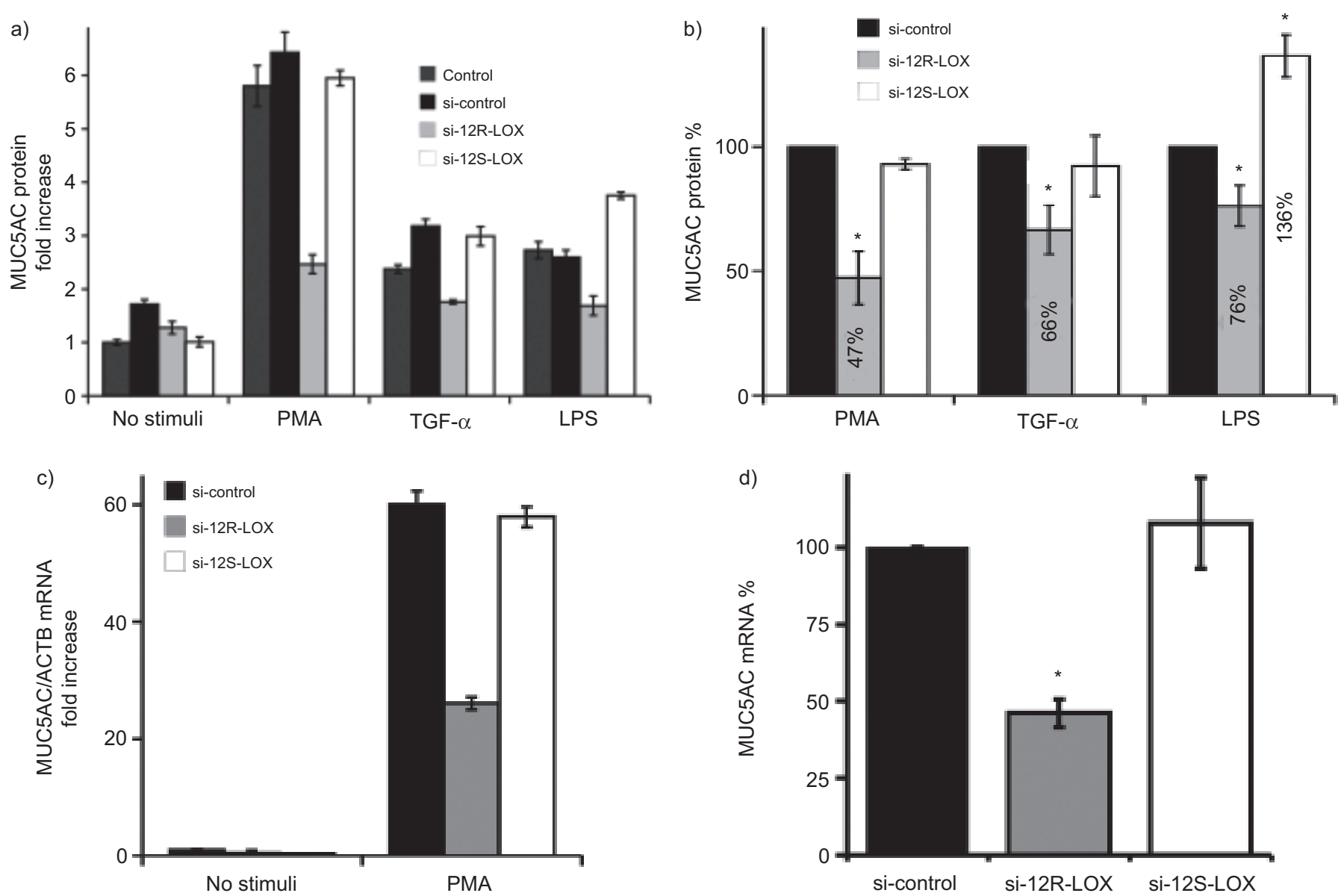

FIGURE 3. Expression of MUC5AC in 12-lipoxygenase (LOX) silenced NCI-H292 cells. Cells transfected with siRNA were stimulated with phorbol myristate acetate (PMA) (20 nM), transforming growth factor (TGF)- $\alpha\left(20 \mathrm{ng} \cdot \mathrm{mL}^{-1}\right)$ or lipopolysaccharide (LPS) $\left(10 \mu \mathrm{g} \cdot \mathrm{mL}^{-1}\right)$ for $24 \mathrm{~h}$. Then, MUC5AC protein was measured in cell lysates as fold increase referred to a) non-stimulated/non-transfected cells (control) (arbitrary units=1) or b) referred to stimulated/control si-RNA (si-control)-transfected cells (arbitrary value $=100 \%$ ). c) mRNA was measured and -fold increase expressed considering non-stimulated/si-control-transfected cells (arbitrary units=1). d) \% MUC5AC expression in PMA-stimulated cells is referred to si-control transfected cells (100\%). Mean \pm SD of triplicates are shown from one representative experiment of a total of three in a) and c). In b) and d) mean \pm SD of triplicates from three independent experiments are shown. ${ }^{*}: p<0.05$ versus cells transfected with si-control and stimulated with the corresponding stimulus.

(242-A01; Abnova, Taipei, Taiwan), mouse anti-human $\beta$-actin antibody (A5316; Sigma, Saint-Quentin Fallavier, France) and the corresponding secondary antibodies (anti-mouse IgG for 12R-LOX and $\beta$-actin primary antibodies or anti-rabbit IgG for anti-phospho-p44/42 primary antibody) HRP-labeled. ECL (GE Healthcare, Saclay-Orsay, France) was used as chemiluminescence detection kit.

\section{Electrophoretic mobility shift assay}

Nuclear proteins were extracted from $2 \times 10^{6}$ cells as described previously [27]. The double-stranded oligonucleotide containing a Sp1 binding site consensus sequence (5'-attcgatcggggcggggcgagc- $\left.3^{\prime}\right)$ was labelled with $\left[\gamma^{-32} \mathrm{P}\right] \mathrm{dATP}\left(3,000 \mathrm{Ci} \cdot \mathrm{mmol}^{-1}\right.$ at $10 \mathrm{mCi} \cdot \mathrm{mL}^{-1}$ ) and purified using a ProbeQuant G-50 micro column (GE Healthcare). The following steps were performed as described by IGNATOV and KEATH [28]. Binding reactions were performed in $40 \mathrm{mM}$ hydroxyethyl piperazine ethane sulphonic acid (HEPES), pH 7.6, $10 \mathrm{mM} \mathrm{NaCl}, 1.5 \mathrm{mM} \mathrm{MgCl}_{2}, 2 \%$ glycerol, containing $1 \mu \mathrm{g}$ of poly(dI-dC), $0.8 \mu \mathrm{Ci}$ of labeled oligonucleotide (1 ng) and $5 \mu \mathrm{g}$ of nuclear extract. The binding specificity was assessed with 50-fold excess cold specific (Sp1 consensus) or nonspecific oligonucleotide (peroxisome proliferator-activated receptors (PPAR) consensus).

\section{Alcian blue staining}

NCI-H292 cells were fixed (formalin 10\%, $10 \mathrm{~min}$ ) and stained with Alcian blue colorant in acetic acid ( $\mathrm{pH} 2.5)$ for $30 \mathrm{~min}$ at room temperature. Cells were counterstained with saphranine for nuclei observation, mounted and observed in a Nikon E 800 microscope. Pictures were acquired using a Nikon digital still DXM 1200 camera controlled by ACT-1 software version 2 (Nikon, Champigny sur Marne, France).

\section{Cell viability}

Lactate dehydrogenase (LDH) activity released from cells was measured with the CytoTox 96 Nonradioactive Cytotoxicity assay (Promega).

\section{Statistical analysis}

Data are presented as mean $\pm \mathrm{SD}$. ANOVA was used to determine statistically significant differences among groups followed by Student-Newman-Keuls multiple range test for paired comparisons. 
When indicated, unpaired t-test was used to compare two groups. $\mathrm{p}<0.05$ was considered significant.

\section{RESULTS}

\section{Involvement of the LOX pathway in PMA-induced MUC5AC production}

We aimed at identifying AA metabolites and signalling pathways involved in MUC5AC expression. For this purpose, we examined MUC5AC levels in supernatants and cell lysates of PMA-stimulated NCI-H292 cells, in the presence of specific COX or LOX inhibitors. Only results from cell lysates are presented, but similar findings were observed on supernatants. We examined the effect of two COX inhibitors (aspirin, NS398) and a general LOX inhibitor (NDGA) on MUC5AC expression. NDGA significantly reduced PMA-induced MUC5AC expression, whereas COX inhibitors had no effect (fig. 1a). We verified that COX inhibitors efficiently reduced $\mathrm{PGE}_{2}$ release (fig. 1b). These data indicate that the LOX but not the COX pathway is involved in MUC5AC expression in PMA-stimulated cells.

\section{Role of 12-LOX in MUC5AC production}

Using more selective LOX inhibitors, we observed that only those targeting 12-LOX (CDC, baicalein) but not those targeting 5-LOX (BW-A4C, zileuton) or 15-LOX inhibitors (PD-146176), reduced PMA-induced MUC5AC expression (fig. 2a). In contrast, neither CDC nor baicalein reduced IL-8 secretion induced by PMA (data not shown). Furthermore, both baicalein and CDC decreased significantly PMA-induced MUC5AC mRNA expression (fig. 2b). Effects of these inhibitors were not due to cell cytotoxicity as measured by LDH release (Materials and methods section). Therefore, these results indicate that 12-LOX modulates MUC5AC expression at a transcriptional level.

\section{R-LOX is involved in MUC5AC expression}

Because NCI-H292 cells express both 12S-LOX and 12R-LOX (see the online supplementary figure), we examined the impact of transcriptional silencing of each 12-LOX form on MUC5AC expression. As fig. 3a shows, silencing of 12R-LOX expression decreased PMA-, TGF- $\alpha$ - and LPS-induced MUC5AC expression. MUC5AC inhibition was more pronounced after PMA treatment $(53 \%)$ compared to TGF- $\alpha(34 \%)$ and LPS treatment (24\%) (fig. 3b). By contrast, 12S-LOX silencing had no effect on either PMA- or TGF- $\alpha$-induced MUC5AC (fig. 3a and b), though, surprisingly, it significantly enhanced LPS-induced MUC5AC production (fig. 3a and b). Next, we analysed the effect of 12-LOX silencing on MUC5AC mRNA expression in PMA-stimulated cells. Silencing of 12R-LOX, but not that of 12S-LOX, reduced MUC5AC mRNA expression (fig. 3c and d). Interestingly, 12R-LOX siRNA did not modify PMA-induced IL-8 secretion (fig. $4 a$ and $b$ ). Taken together these data show a role for 12R-LOX in the induction of MUC5AC expression.

\section{2-LOX inhibitors reduce PMA-induced ERK and Sp1 activation}

In NCI-H292 cells, activation of PKC by PMA activates ERK and translocates Sp1 which binds to MUC5AC promoter to initiate transcription [7, 9]. As shown in fig. 5a, CDC and baicalein reduced PMA-induced ERK phosphorylation. Moreover, electrophoretic mobility shift assay (EMSA) analyses showed an enhanced Sp1 translocation in PMA stimulated cells (fig. 5b). Conversely, baicalein reduced the Sp1-shifted band (fig. 5b).
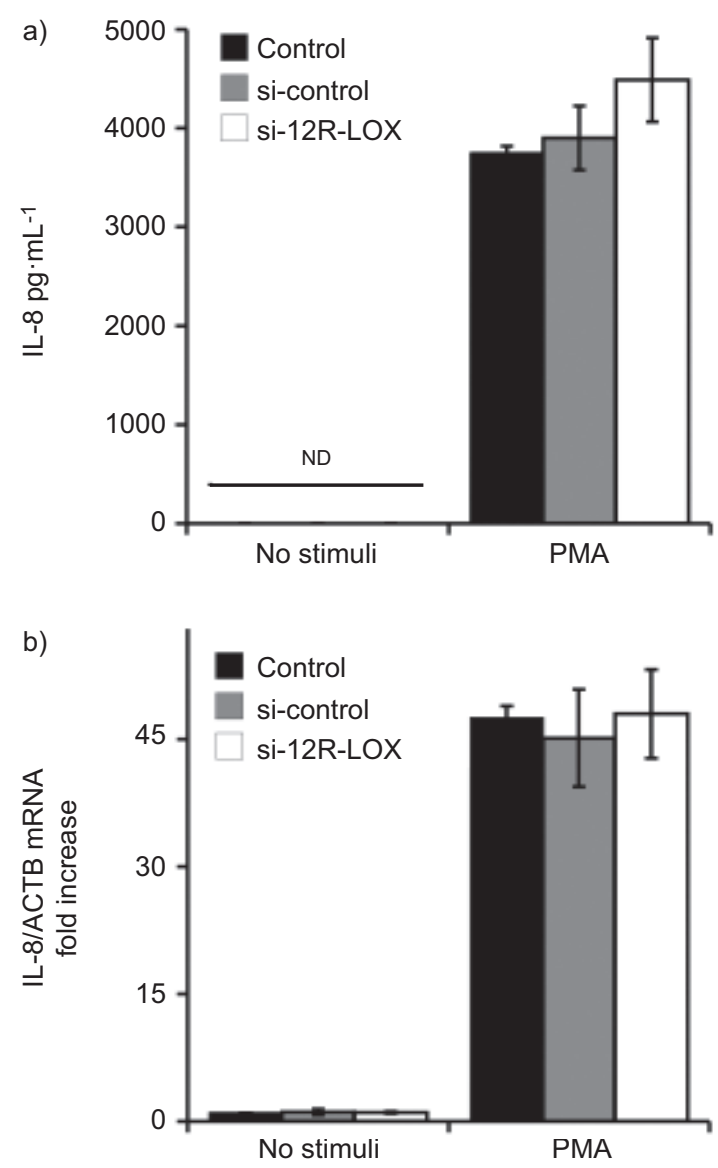

FIGURE 4. Expression of interleukin (IL)-8 induced by phorbol myristate acetate (PMA) in 12R-lipoxygenase (LOX) silenced NCl-H292. Cells transfected with 12R-LOX siRNAs (100 nM) were stimulated with PMA (20 nM) for $24 \mathrm{~h}$. Then, IL-8 protein was measured in cell supernatants by a) sandwich ELISA and b) IL-8 phorbol myristate acetate mRNA expression by real time RT-PCR. Means \pm SD of triplicates are shown from one representative experiment of a total of three. ND: not detected; si-control: nontargeting si-RNA provided by manufacturer.

Similar results were obtained with the other 12-LOX inhibitor, CDC (data not shown). Taken together, these data suggest that 12-LOX plays a role in PMA-induced ERK-Sp1 activation.

\section{2(R)-HETE upregulates MUC5AC expression and activates ERK-Sp1 signalling pathways}

12(R)-HETE, a 12R-LOX product, induced MUC5AC protein in a dose-dependent manner, with a maximal effect at $8 \mu \mathrm{M}$ (fig. 6a). Furthermore, 12(R)-HETE was able to activate ERK (fig. 6b), whilst the MEK1/2 (ERK kinase) inhibitor (PD98059) blocked 12(R)-HETE-induced ERK phosphorylation (fig. 6b). In addition, 12(R)-HETE induced Sp1 translocation with a peak of activity at $2 \mathrm{~h}$ after stimulation (fig. $6 \mathrm{c}$ ). These findings indicate that 12(R)-HETE stimulates the ERK-Sp1-signalling pathway.

\section{R-LOX also regulates other respiratory mucins}

We examined whether 12R-LOX also modulates PMA-induced MUC5B and MUC2 expression. Silencing of 12R-LOX reduced MUC5B and MUC2 expression by $28 \%$ and 35\%, respectively (fig. 7a). These effects were less pronounced than those observed on MUC5AC (53\% inhibition) (fig. 7a). Finally, we 
a)
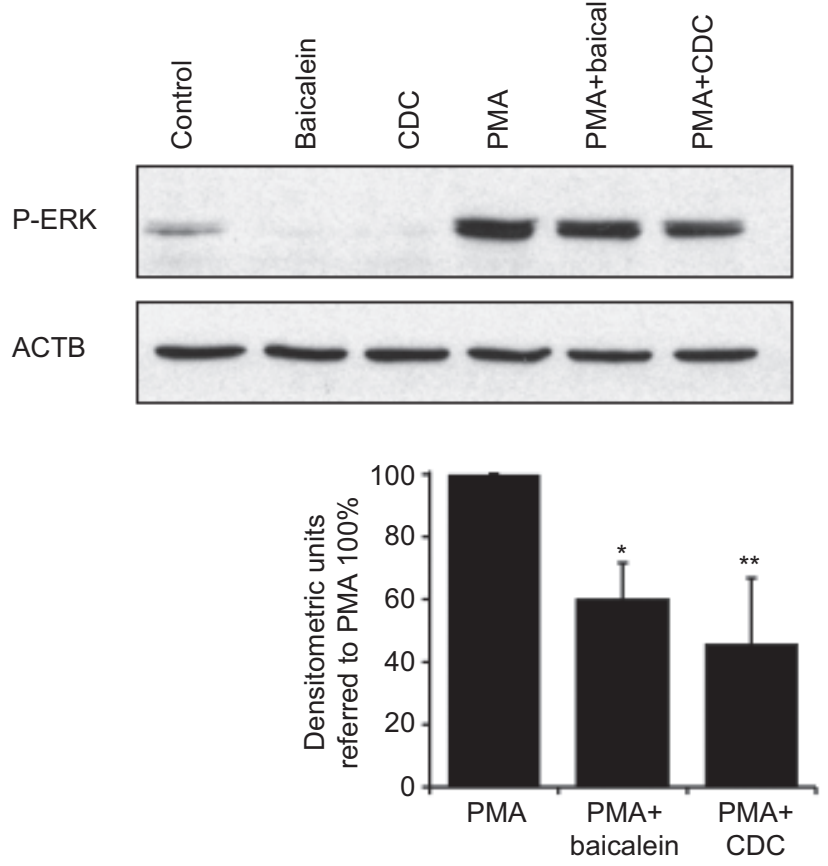

b)

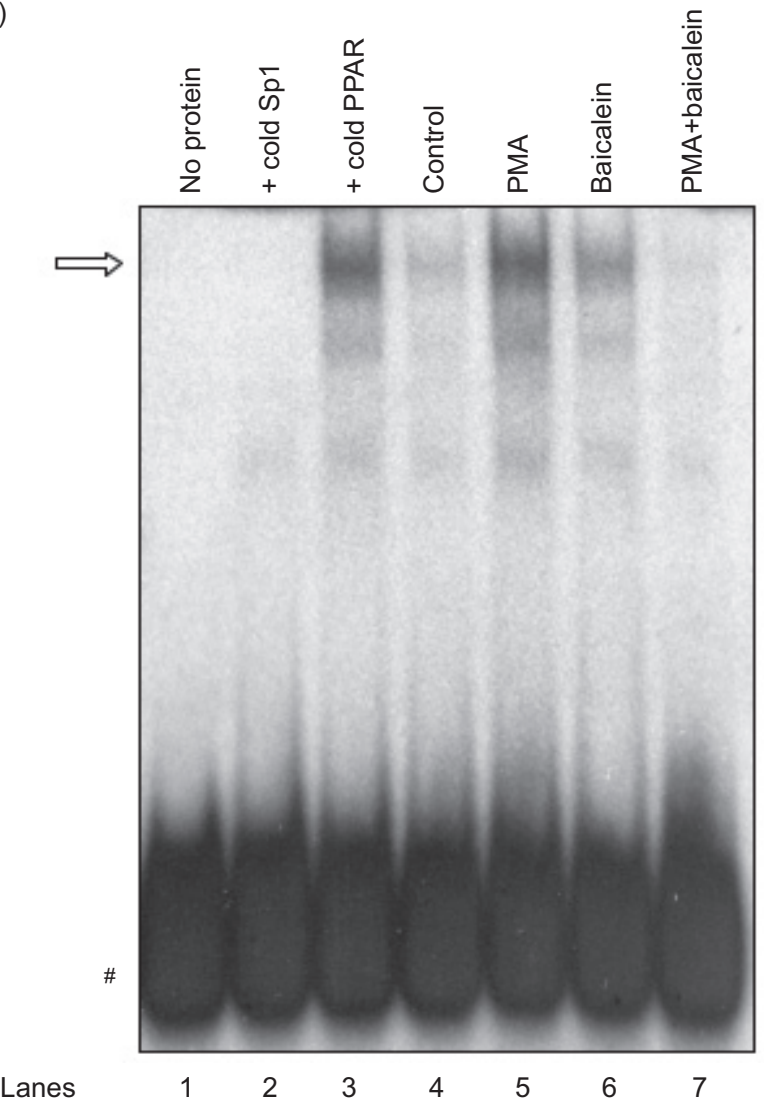

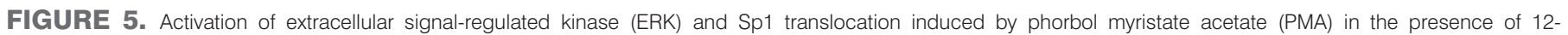

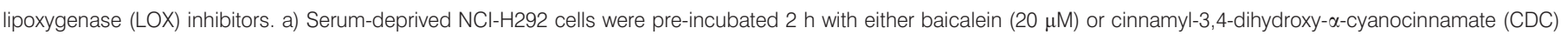

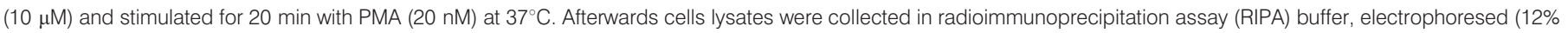

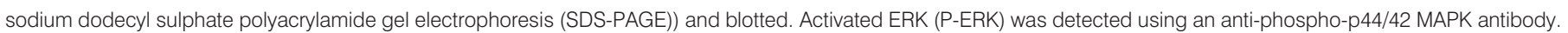

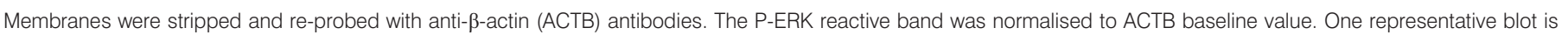

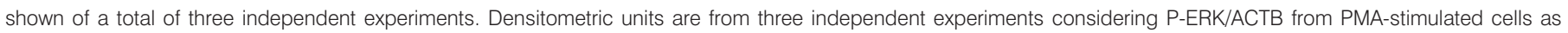

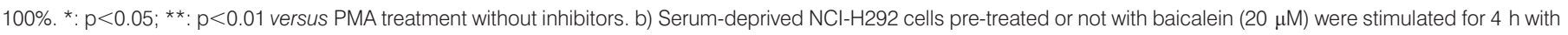

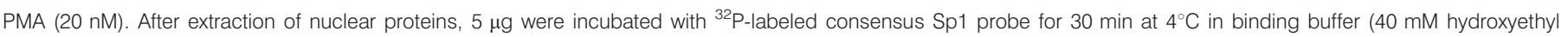

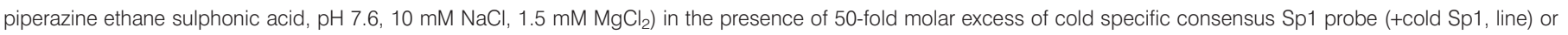

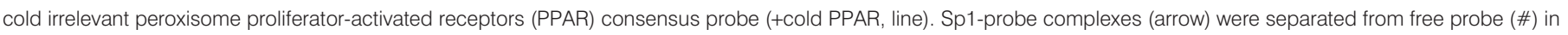

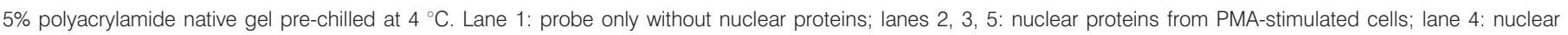

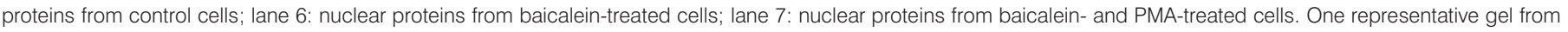
three independent experiments is shown.

investigated the role of 12-LOX on the production of whole mucus by NCI-H292 cells. This was performed using Alcian blue $(\mathrm{AB})$ staining on cell monolayers in the presence or absence of baicalein. Increased $A B$ staining was observed in cells stimulated with PMA compared to non-stimulated cells (fig. $7 \mathrm{~b}$ ), reflecting production of mucus induced by PMA. Baicalein strongly reduced this staining (fig. $7 \mathrm{~b}$ ), a finding confirmed when cells were treated with CDC (data not shown).

\section{2-LOX regulates respiratory mucins in primary human bronchial epithelial cells}

To examine the role of 12-LOX in the expression of respiratory mucins in primary cells, human bronchial epithelial cells were growth in air-liquid interface and stimulated with supernatants of $P$. aeruginosa, a pathophysiological stimulus in $\mathrm{CF}$ patients. $P$. aeruginosa supernatants induced MUC5AC and
MUC2 (fig. 8a and 8b) but not MUC5B expression (fig. 8c). The presence of baicalein reduced $46 \%$ and $36 \%$ of the $P$. aeruginosa-induced expression of MUC5AC and MUC2, respectively (fig. 8a and $8 b$ ).

\section{DISCUSSION}

In the present work, performed on the NCI-H292 cell line and primary bronchial epithelial cells, we demonstrate a key role for 12-LOX in mucin MUC5AC expression. We have used PMA as a mucin inducer because previous studies have shown that expression of MUC5AC induced by this drug is dependent of EGFR activation, a key receptor controlling mucin production in the airways [10]. EGFR-neutralising antibodies or inhibitors of EGFR phosphorylation blocked PMA-induced MUC5AC expression [9]. In selected experiments more physiological stimuli (TGF- $\alpha$, LPS, supernatants of $P$. aeruginosa) have been used to 
a)

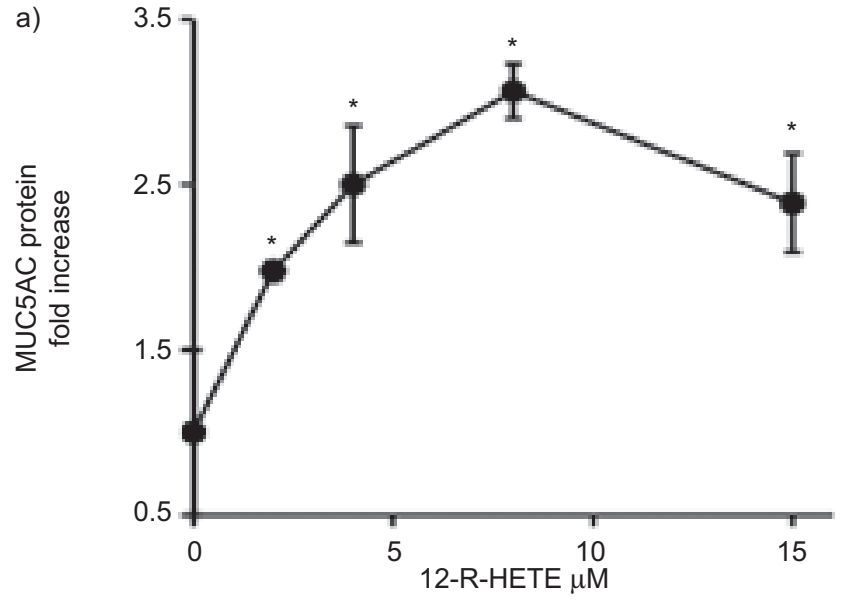

b)

\begin{tabular}{|c|c|c|c|c|c|c|}
\hline PMA & - & + & + & - & - & - \\
\hline PD98059 & - & - & + & - & + & - \\
\hline 12(R)-HETE & - & - & - & + & + & ++ \\
\hline P-ERK & & & & $=$ & & \\
ACTB & & & & & & \\
\cline { 2 - 7 } & & & & & & \\
\hline
\end{tabular}

c)

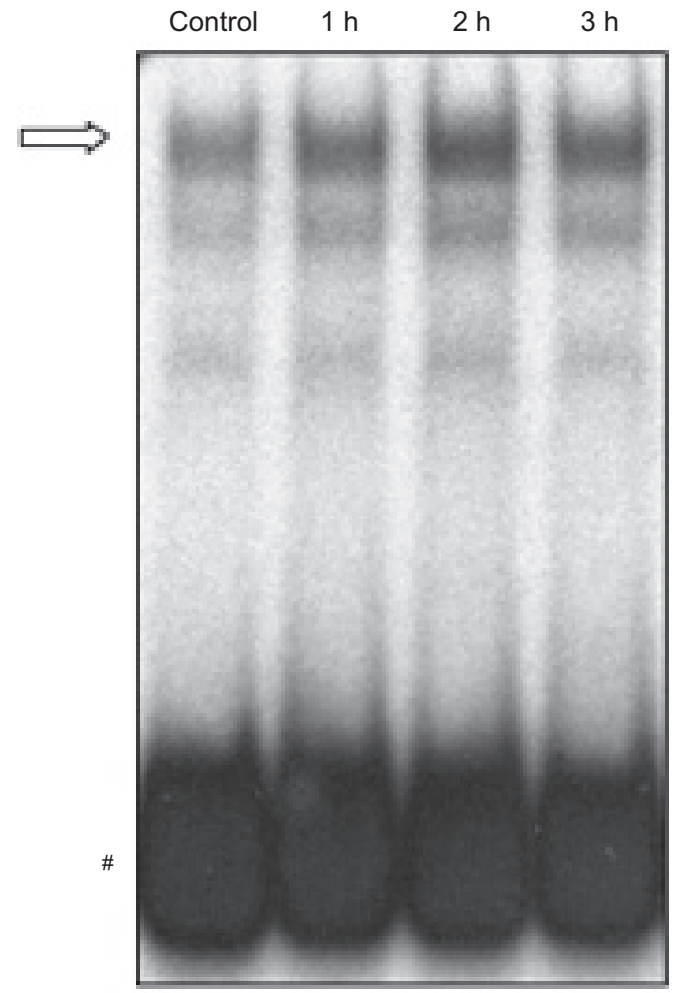

FIGURE 6. 12(R)-hydroxyeicosatetraenoic acid (HETE) induces MUC5AC expression, extracellular signal-regulated kinase (ERK) activation and Sp1 translocation in $\mathrm{NCl}-\mathrm{H} 292$. a) 12(R)-HETE or vehicle were added to cells. After $24 \mathrm{~h}$ of culture, MUC5AC protein was measured in cell lysates by ELISA. Mean \pm SD is represented as -fold increase normalised to MUC5AC protein level in basal conditions from three independent experiments performed in triplicates. *: $p<0.05$ versus nonstimulated cells (t-test). b) ERK phosphorylation (P-ERK) was analysed as described in figure $5 a$ after 20 min of incubation with phorbol myristate acetate (PMA) (20 nM), PD98059 (20 $\mu$ M), $12(\mathrm{R})$ HETE $(2 \mu \mathrm{M}(+)$ or $8 \mu \mathrm{M}(++))$ or the combinations indicated in the table. PD98059 was added also 30 min before either PMA or 12(R)-HETE. c) Cells were treated with 12(R)HETE $(8 \mu \mathrm{M})$ at indicated times (control, $\mathrm{t}=0$ ) before performing electrophoretic mobility shift assay as described in fig. $5 \mathrm{~b}$.

demonstrate the involvement of 12-LOX in mucin expression. Downstream signalling pathways after EGFR activation by PMA conducting to MUC5AC expression have been partially characterised [4, 7], but the role of 12-LOX in these pathways has not been explored.

Although active COX enzymes are present in NCI-H292 cells, COX inhibitors had no effect on PMA-induced MUC5AC expression. Previous works have shown a role of COX in MUC5AC expression [15, 16]. However, it should be noted that in these reports the authors used IL-1 $\beta$ as MUC5AC inducer instead of PMA. IL-1 $\beta$ signalling pathways leading to mucin expression differ from those for PMA. PMA induces MUC5AC expression via matrix metalloprotease-mediated release of TGF- $\alpha$, which subsequently activates EGFR [29], a mechanism different from that for IL-1 $\beta$ [15]. Moreover, GRAY et al. [15] used a model of tracheobronchial epithelial cells, in which different cell types are present (e.g. ciliated, basal, mucus cells). Therefore, in their model, one possibility is that COX metabolites modulate MUC5AC expression by mucus producing cells via a paracrine mechanism. In contrast, our study was performed on a single cell type, which involves the study of the autocrine regulation of mucin expression.

LOX represents a family of enzymes that play diverse biological roles. Using pharmacological and transcriptional inhibition we explored which LOX is involved in MUC5AC expression. We first examined whether 5-LOX, which leads to leukotriene production, is involved in MUC5AC expression. Indeed, leukotrienes have been shown to induce mucus expression in the lung [11], and human bronchial epithelial cells express an active 5-LOX [30]. Our results showed that this LOX is not involved in MUC5AC expression in our cell system.

A recent study has shown that a 15-LOX-1 inhibitor abrogates MUC5AC expression induced by long-term IL-13 stimulation of bronchial epithelial cells [22]. However, in the present study, using the same 15-LOX-1 inhibitor reported in the study by ZHAO et al. [22], we found that this enzyme is not involved in PMA-induced MUC5AC expression. We also observed that the 15-LOX-1 inhibitor did not reduce TGF- $\alpha$-induced MUC5AC expression (data not shown). Here again, differences in the signalling pathways activating MUC5AC expression (IL-13 receptor-dependent versus EGFR-dependent pathways) and duration of the stimulation might explain these discrepancies between our findings and those of ZHAO et al. [22].

Our pharmacological approach showed that $12-\mathrm{LOX}$ is the only LOX that plays a role in MUC5AC expression in NCI-H292 cells. Because two 12-LOX (12R-LOX and 12S-LOX) encoded by distinct genes are present in mammalian cells, we used a siRNA strategy to identify which form is involved in mucus 
a) $\square$ Control siRNA

12R-LOX siRNA

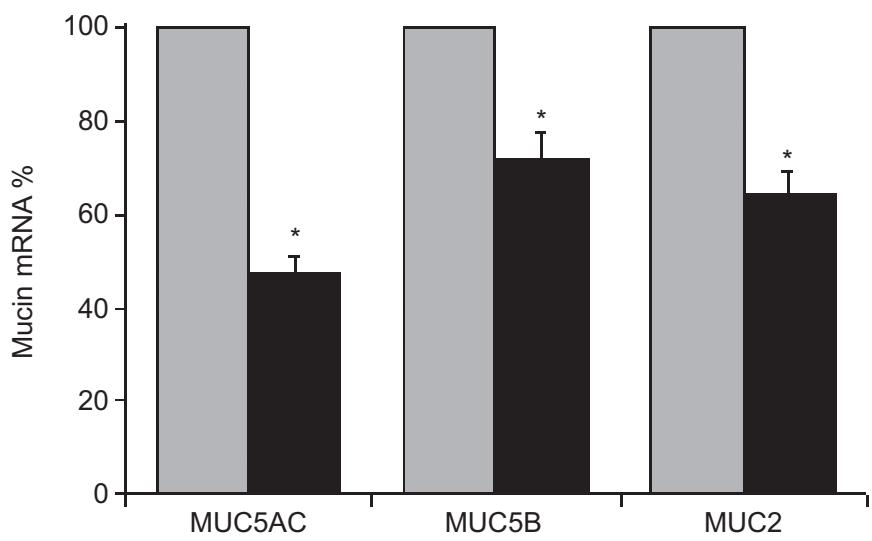

b)
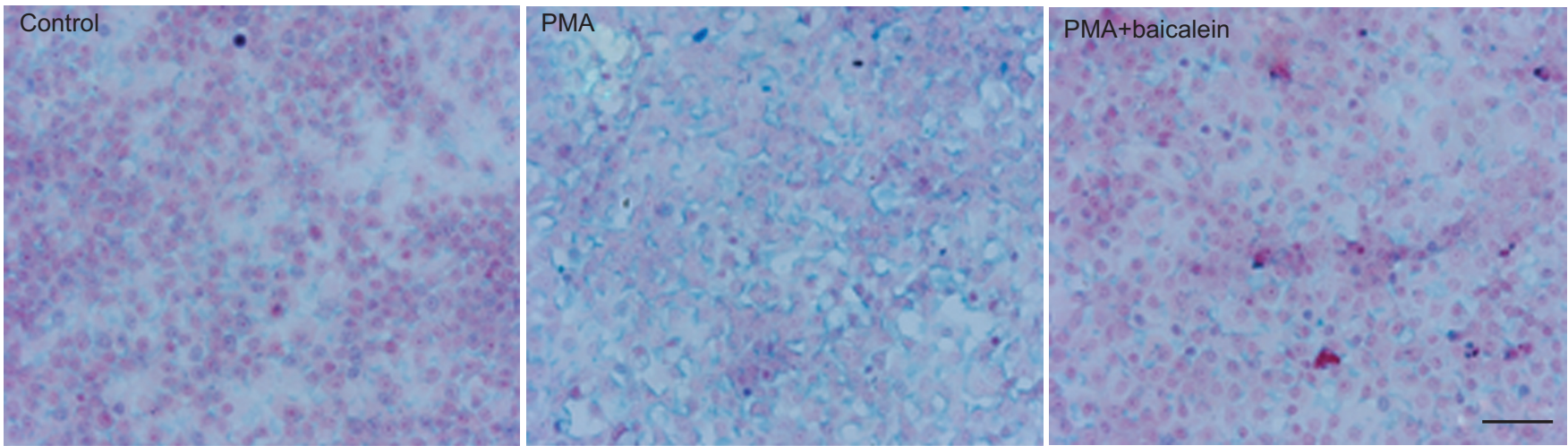

FIGURE 7. Modulation of airway mucins and mucus by 12R-lipoxygenase (LOX). a) Real-time PCR from phorbol myristate acetate (PMA)-stimulated cells transfected with 12R-LOX siRNA or control siRNA, was performed with specific MUC5AC, MUC5B or MUC2 primers as described in the Materials and methods section. Levels of expression were normalised to PMA-stimulated cells transfected with control siRNA (100\%). Mean \pm SD of triplicates from three independent experiments are represented. *: $\mathrm{p}<0.05$ versus si-control transfected cells. b) Cells were first pre-incubated or not $1 \mathrm{~h}$ with baicalein $(20 \mu \mathrm{M})$ and then stimulated or not for $24 \mathrm{~h}$ with PMA (20 nM). Cells were then fixed and stained with Alcian blue (acidic mucins in blue) and saphranine (nuclei in red). Scale bar $=100 \mu$ m. Representative images from one out of two independent experiments are shown. Treatment with cinnamyl-3,4-dihydroxy- $\alpha$-cyanocinnamate $(10 \mu \mathrm{M})$ showed the same results (data not shown).
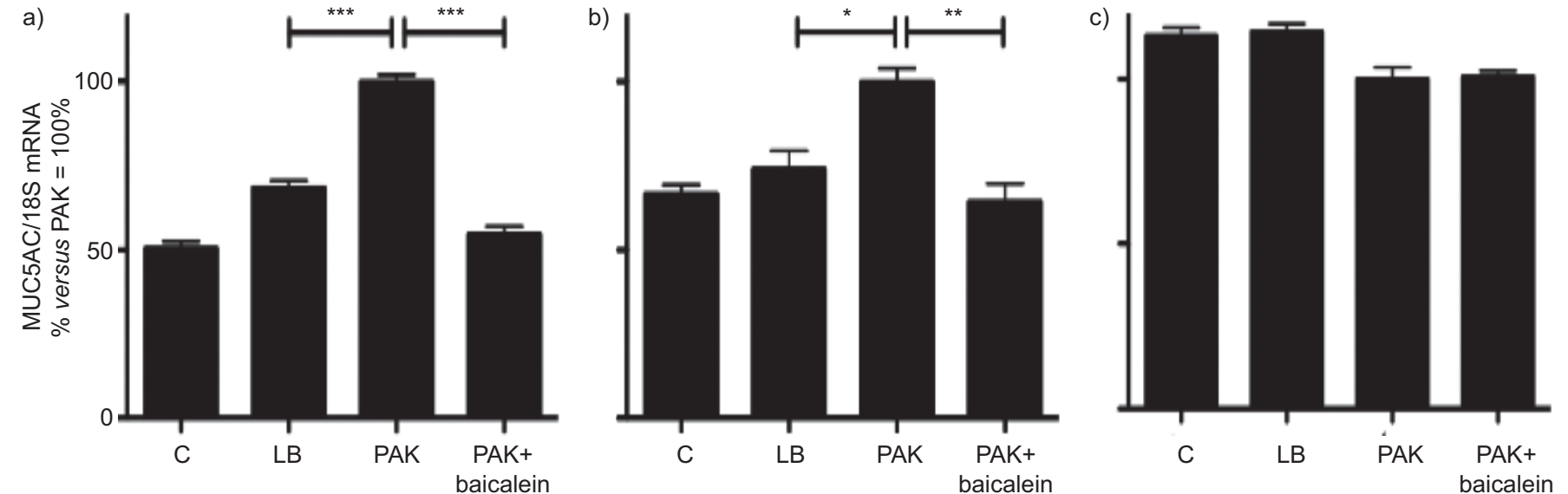

FIGURE 8. Effect of baicalein in Pseudomonas aeruginosa supernatant-induced mucins expression in human primary epithelial cells. The cells were cultured in air-liquid interface and pre-treated with baicalein $(20 \mu \mathrm{M}) 1 \mathrm{~h}$ and during treatment with bacteria $(P$. aeruginosa, strain PAK) supernatants diluted at 1:8 or bacteria culture medium, Luria-Bertani (LB, 1:8) for $24 \mathrm{~h}$ as indicated in the Materials and methods. Total mRNA was prepared and subjected to real-time quantitative PCR to measure a) MUC5AC, b) MUC2 and c) MUC5B levels. The results show the percentage of inhibition of mucins mRNA expression by baicalein compared to cells treated with PAK supernatant alone. Values represent mean $\pm S D$ of three independent experiments from three different donors. $*: p<0.05 ; * \star: p<0.01 ; * \star *: p<0.001$. 
production in our cell system. Our results showed that PMAinduced MUC5AC expression is reduced by 12R-LOX but not by 12S-LOX siRNA. Interestingly, using more physiological stimuli, TGF- $\alpha$ and LPS (ligands for EGFR and TLR-4 receptor respectively, two key receptors in lung pathophysiology), we demonstrate a stimulating role of 12R-LOX in TGF- $\alpha$ - and LPSinduced MUC5AC expression. We also showed that 12R-LOX plays a role in the modulation of MUC5B and MUC2 expression and in the production of whole mucus by NCIH292 monolayers induced by PMA. Moreover, induction of mucins by $P$. aeruginosa supernatants, a pathogen related to lung dysfunction in CF, was reduced by a 12-LOX inhibitor in primary bronchial epithelial cells. Altogether, our data suggest a role of 12-LOX in the expression of mucins relevant to the pathogenesis of lung obstructive diseases.

Trans-activation of EGFR by PMA [9] is known to induce ERK activation and binding of the transcription factor $\mathrm{Sp} 1$ to the MUC5AC promoter [4, 7]. Here, we show that 12R-LOX siRNA inhibits TGF- $\alpha$-induced MUC5AC expression and that 12-LOX inhibitors reduce ERK activation and Sp1 translocation induced by PMA. Altogether, these results suggest that 12R-LOX, but not 12S-LOX, mediates PMA-induced MUC5AC expression via an EGFR-related signalling pathway involving ERK- and Sp1 activation.

We next examined whether 12(R)-HETE, one product of 12RLOX, mediates MUC5AC expression in NCI-H292 cells. We show that addition of 12R-HETE stimulates MUC5AC expression, ERK activation and Sp1 translocation. However, the effects of 12R-HETE were less pronounced than those of PMA in terms of MUC5AC production. This could be explained thus: 1) 12RLOX independent pathways are involved in PMA-induced MUC5AC expression; 2) other metabolites derived (directly or indirectly) from $12 \mathrm{R}$-LOX activity might also participate in the process. Regarding the mechanism through which 12(R)-HETE induces MUC5AC, 12(R)-HETE is a potent activator of the arylhydrocarbon receptor (AhR) [31], a ligand-regulated transcription factor related to environmental insults. Activation of AhR receptor results in upregulation of MUC5AC expression in mouse lungs and in NCI-H292 cells [32, 33]. Therefore, 12R-LOX stimulating action could be explained by the capacity of $12 \mathrm{R}$ LOX products (e.g. 12(R)-HETE) to activate AhR.

In conclusion, this study demonstrates that 12R-LOX, an enzyme that produces AA metabolites with R-chirality, mediates mucus production by mucus-producing cells, a main feature of pulmonary obstructive diseases. Therefore, 12R-LOX represents a suitable new target for therapeutic intervention to reduce mucus production in these pathologies. Moreover, bronchioloalveolar carcinoma occurs with excessive production of sputum associated to overexpression of respiratory gel forming mucins. Therapies preventing EGFR activation have shown beneficial effects in these patients [34]. The role of 12-LOX in EGFR-dependent mucin production suggests 12-LOX inhibitors as potential agents for the treatment of bronchioloalveolar carcinoma.

\section{SUPPORT STATEMENT}

The work was supported by the French cystic fibrosis association "Vaincre la Mucoviscidose" (VLM) and the foundation "Legs Poix". I. Garcia-Verdugo was supported by VLM.

\section{STATEMENT OF INTEREST}

None declared.

\section{ACKNOWLEDGEMENTS}

The authors thank M. Huerre and P. Ave (Pasteur Institut, Paris, France) for their help and advice during mucus staining, P-R. Burgel (Cochin Hospital, Paris, France) for his advice in MUC5AC ELISA and P. Krieg (German Cancer Research Center, Heidelberg, Germany) for his fruitful discussions about lipoxygenases.

\section{REFERENCES}

1 Rose MC, Voynow JA. Respiratory tract mucin genes and mucin glycoproteins in health and disease. Physiol Rev 2006; 86: 245-278.

2 Audie JP, Janin A, Porchet N, et al. Expression of human mucin genes in respiratory, digestive, and reproductive tracts ascertained by in situ hybridization. J Histochem Cytochem 1993; 41: 1479-1485.

3 Pigny P, Guyonnet-Duperat V, Hill AS, et al. Human mucin genes assigned to 11p15.5: identification and organization of a cluster of genes. Genomics 1996; 38: 340-352.

4 Perrais M, Pigny $\mathrm{P}$, Copin $\mathrm{MC}$, et al. Induction of $\mathrm{MUC2}$ and MUC5AC mucins by factors of the epidermal growth factor (EGF) family is mediated by EGF receptor/Ras/Raf/extracellular signal-regulated kinase cascade and Sp1. J Biol Chem 2002; 277: 32258-32267.

$5 \mathrm{Wu}$ DY, Wu R, Chen Y, et al. PMA stimulates MUC5B gene expression through an Sp1-based mechanism in airway epithelial cells. Am J Respir Cell Mol Biol 2007; 37: 589-597.

6 Hovenberg HW, Davies JR, Herrmann A, et al. MUC5AC, but not MUC2, is a prominent mucin in respiratory secretions. Glycoconj J 1996; 13: 839-847.

7 Hewson CA, Edbrooke MR, Johnston SL. PMA induces the MUC5AC respiratory mucin in human bronchial epithelial cells, via PKC, EGF/TGF-alpha, Ras/Raf, MEK, ERK and Sp1-dependent mechanisms. J Mol Biol 2004; 344: 683-695.

8 Takeyama K, Dabbagh K, Lee HM, et al. Epidermal growth factor system regulates mucin production in airways. Proc Natl Acad Sci USA 1999; 96: 3081-3086.

9 Shao MX, Ueki IF, Nadel JA. Tumor necrosis factor alphaconverting enzyme mediates MUC5AC mucin expression in cultured human airway epithelial cells. Proc Natl Acad Sci USA 2003; 100: 11618-11623.

10 Burgel PR, Nadel JA. Epidermal growth factor receptor-mediated innate immune responses and their roles in airway diseases. Eur Respir J 2008; 32: 1068-1081.

11 Johnson HG, McNee ML. Regulation of canine mucus secretion by a novel leukotriene synthesis inhibitor (U-60,257). Int Arch Allergy Appl Immunol 1984; 75: 97-101.

12 Marom Z, Shelhamer JH, Kaliner M. Effects of arachidonic acid, monohydroxyeicosatetraenoic acid and prostaglandins on the release of mucous glycoproteins from human airways in vitro. J Clin Invest 1981; 67: 1695-1702.

13 Dif $\mathrm{F}, \mathrm{Wu} \mathrm{YZ}$, Burgel PR, et al. Critical role of cytosolic phospholipase A2 $\alpha$ in bronchial mucus hypersecretion in CFTRdeficient mice. Eur Respir J 2010; 36: 1120-1130.

14 Rich B, Peatfield AC, Williams IP, et al. Effects of prostaglandins $\mathrm{E} 1, \mathrm{E} 2$, and F2 alpha on mucin secretion from human bronchi in vitro. Thorax 1984; 39: 420-423.

15 Gray T, Nettesheim P, Loftin C, et al. Interleukin-1beta-induced mucin production in human airway epithelium is mediated by cyclooxygenase-2, prostaglandin E2 receptors, and cyclic AMPprotein kinase A signaling. Mol Pharmacol 2004; 66: 337-346.

16 Kim YD, Kwon EJ, Park DW, et al. Interleukin-1beta induces MUC2 and MUC5AC synthesis through cyclooxygenase-2 in NCIH292 cells. Mol Pharmacol 2002; 62: 1112-1118. 
17 Brash AR. Lipoxygenases: occurrence, functions, catalysis, and acquisition of substrate. J Biol Chem 1999; 274: 23679-23682.

18 Lagarde M, Calzada C, Zakaroff A, et al. Biological relevance of the 12-lipoxygenase pathway for platelet and lymphocyte functions. Adv Exp Med Biol 1999; 447: 87-93.

19 Owens JM, Shroyer KR, Kingdom TT. Expression of cyclooxygenase and lipoxygenase enzymes in sinonasal mucosa of patients with cystic fibrosis. Arch Otolaryngol Head Neck Surg 2008; 134: 825-831.

20 Epp N, Furstenberger G, Muller K, et al. 12R-lipoxygenase deficiency disrupts epidermal barrier function. J Cell Biol 2007; 177: 173-182.

21 Kuhn H, O'Donnell VB. Inflammation and immune regulation by 12/15-lipoxygenases. Prog Lipid Res 2006; 45: 334-356.

22 Zhao J, Maskrey B, Balzar S, et al. Interleukin-13-induced MUC5AC is regulated by 15-lipoxygenase 1 pathway in human bronchial epithelial cells. Am J Respir Crit Care Med 2009; 179: 782-790.

23 Burgel PR, Lazarus SC, Tam DC, et al. Human eosinophils induce mucin production in airway epithelial cells via epidermal growth factor receptor activation. J Immunol 2001; 167: 5948-5954.

24 Lidell ME, Bara J, Hansson GC. Mapping of the 45M1 epitope to the C-terminal cysteine-rich part of the human MUC5AC mucin. FEBS J 2008; 275: 481-489.

25 Finzi L, Barbu V, Burgel PR, et al. MUC5AC, a gel-forming mucin accumulating in gallstone disease, is overproduced via an epidermal growth factor receptor pathway in the human gallbladder. Am J Pathol 2006; 169: 2031-2041.

26 Nigam S, Zafiriou MP, Deva R, et al. Hepoxilin A3 (HXA3) synthase deficiency is causative of a novel ichthyosis form. FEBS Lett 2008; 582: 279-285.
27 Alaoui-El-Azher $\mathrm{M}, \mathrm{Wu} \mathrm{Y}$, Havet $\mathrm{N}$, et al. Arachidonic acid differentially affects basal and lipopolysaccharide-induced sPLA(2)IIA expression in alveolar macrophages through NF-kappaB and PPAR-gamma-dependent pathways. Mol Pharmacol 2002; 61: 786-794.

28 Ignatov A, Keath EJ. Gel shift assay of nuclear extracts from Histoplasma capsulatum demonstrates the presence of several DNA binding proteins. Infect Immun 2002; 70: 2238-2241.

29 Shao MX, Nadel JA. Dual oxidase 1-dependent MUC5AC mucin expression in cultured human airway epithelial cells. Proc Natl Acad Sci USA 2005; 102: 767-772.

30 James AJ, Penrose JF, Cazaly AM, et al. Human bronchial fibroblasts express the 5-lipoxygenase pathway. Respir Res 2006; 7: 102.

31 Chiaro CR, Patel RD, Perdew GH. 12(R)-Hydroxy-5(Z),8(Z),10(E),14(Z)eicosatetraenoic acid [12(R)-HETE], an arachidonic acid derivative, is an activator of the aryl hydrocarbon receptor. Mol Pharmacol 2008; 74: 1649-1656.

32 Chiba T, Uchi H, Tsuji G, et al. Arylhydrocarbon receptor (AhR) activation in airway epithelial cells induces MUC5AC via reactive oxygen species (ROS) production. Pulm Pharmacol Ther 2011; 24: 133-140.

33 Wong PS, Vogel CF, Kokosinski K, et al. Arylhydrocarbon receptor activation in NCI-H441 cells and C57BL/6 mice: possible mechanisms for lung dysfunction. Am J Respir Cell Mol Biol 2010; 42: 210-217.

34 Kitazaki T, Soda H, Doi S, et al. Gefitinib inhibits MUC5AC synthesis in mucin-secreting non-small cell lung cancer cells. Lung Cancer 2005; 50: 19-24. 